Article

\title{
A Facile Synthesis for Novel Loperamide Analogs as Potential $\mu$ Opioid Receptor Agonists
}

\author{
Xiaofeng Bao ${ }^{\dagger} *$, Duliang Liu ${ }^{\dagger}$, Yanyan Jin and Yao Yang \\ Department of Biochemical Engineering, Nanjing University Science \& Technology, \\ Chemical Engineering Building B302, 200 Xiaolinwei, Nanjing 210094, China; \\ E-Mails:1d112358@hotmail.com (D.L.); j.yy.1002c@163.com (Y.J.); bxfeng11@hotmail.com (Y.Y.) \\ $\dagger$ These authors contributed equally to this work. \\ * Author to whom correspondence should be addressed; E-Mail: baoxiaofeng@njust.edu.cn; \\ Tel./Fax: +86-25-8431-5945.
}

Received: 6 November 2012; in revised form: 27 November 2012 / Accepted: 28 November 2012 / Published: 3 December 2012

\begin{abstract}
A facile synthesis for novel loperamide analogs as potential $\mu$ opioid receptors is described. The synthetic procedure for compound 5, which contains two 4-phenyl piperidine scaffolds, was optimized, and this compound was synthesized in excellent yield. We also describe a mild and highly efficient protocol for the synthesis of compounds 6 and 7.
\end{abstract}

Keywords: loperamide analogs; $\mu$ opioid receptor agonist; morphine; opioid ligand receptors

\section{Introduction}

Opioid ligand receptors are involved in various physiological activities, including analgesia, miosis, bradycardia, general sedation, hypothermia, insensitivity and depression of the flexor reflexes and have been widely used in medicine, most prominently in the treatment of pain [1,2]. Three separate receptors - $\mu(\mathrm{mu}), \delta$ (delta), and $\kappa$ (kappa) —were proven to be the basis of the pharmacologic responses using in vitro radioligand binding affinity assays and in vivo localization of labeled drug in tissue homogenates or sections $[3,4]$. These reports indicate that $\mu$ opioid receptor (MOR) agonists are useful analgesics in the periphery, especially for inflamed tissues [5,6]. Phenylpiperidine structures, such as loperamide (1) [7,8], $N$-desmethylloperamide (2) [9], diphenoxylate (3) [10], and 4 (Figure 1) [11], are typical representatives of these compounds. Although diphenoxylate and loperamide are MOR 
agonists and show affinity and selectivity for the cloned $\mu$ human opioid receptor, they do not easily pass through the blood-brain barrier (BBB) [12], therefore, they are currently mainly used as antidiarrheal drugs.

Figure 1. Structures of representative MOR agonists.

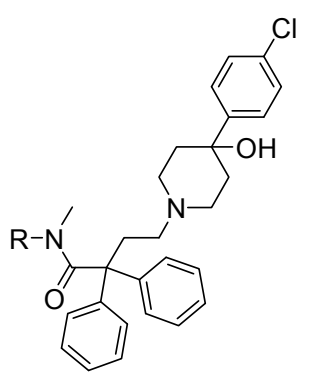

1, R=Me, Loperamide

2, $\mathrm{R}=\mathrm{H}, \mathrm{N}$-desmethylloperamide

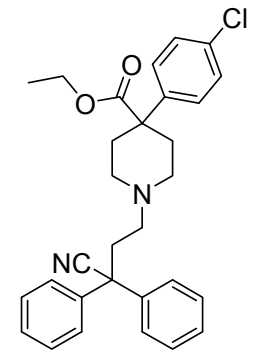

3, Diphenoxylate<smiles>OC1(c2ccc(Cl)cc2)CCN(CCC(c2ccccc2)c2ccccc2)CC1</smiles>

4, 4-(4-chlorophenyl)-1-(3,3diphenylpropyl)piperidin-4-ol

More recently, MOR agonists based on the 4-phenylpiperidine scaffold have been reported and have shown promising results [13,14]. The therapeutic potential of loperamide and other compounds based on 4-phenylpiperidine scaffolds may also be of interest to the scientific community in light of the recent discovery of DiPOA, a novel, systemically available and peripherally restricted MOR agonist with antihyperalgesic activity [15].

Therefore, the compounds 1,4-bis(4-(4-chlorophenyl)-4-hydroxypiperidin-1-yl)-2,2-diphenylbutan1-one (5, Figure 2), 4-(4-(4-fluorophenyl)-4-hydroxypiperidin-1-yl)-2,2-diphenylbutanamide (6, Figure 2) and (Z)-2-(2-(4-(4-fluorophenyl)-4-hydroxypiperidin-1-yl) ethyl)-3-methylene-2-phenylhex-4-enamide (7, Figure 2), which are based on loperamide and diphenoxylate, were designed and synthesized as potential MOR agonists. The novel analog 5 contains two 4-phenylpiperidine scaffolds, and analogs 6 and 7 each contain one 4-phenylpiperidine scaffold. We consider these analogs to be promising MOR agonists.

Figure 2. Structures of novel loperamide analogs.

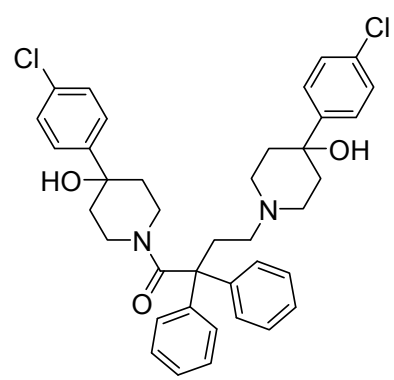

5, 1,4-bis(4-(4-chlorophenyl)-4-hydroxy piperidin-1-yl)-2,2-diphenylbutan-1-one

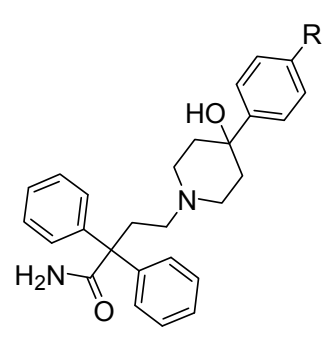

6, $\mathrm{R}=\mathrm{F}$

7, $\mathrm{R}=\mathrm{Br}$ 


\section{Results and Discussion}

As a consequence of our interest in MORs containing 4-phenylpiperidine scaffolds, we developed a mild and highly efficient protocol for the synthesis of compounds 5, $\mathbf{6}$ and 7. We initiated our studies by preparing the key intermediate $\mathbf{1 0 b}$ by coupling commercially available compounds $\mathbf{8}$ and $\mathbf{9 b}$ to achieve product $\mathbf{1 0 b}$ under different reaction conditions, as shown in Table 1. The reaction using conditions based on those of a similar reaction that was previously reported in the literature was not successful for an unknown reason [16]. When the reaction time was prolonged to $30 \mathrm{~h}$ with 3 eq. of DIPEA at $105{ }^{\circ} \mathrm{C}$, we successfully isolated compound $\mathbf{1 0 b}$ in a disappointing $6 \%$ yield. The possible reasons for failure may be that the hydroxyl group on compound $\mathbf{9 b}$ was affected. This group is also a strong nucleophile under basic conditions, as observed in our previously published study [17], and the carboxylates present under basic conditions may also compete with the secondary amine in the nucleophilic substitution reaction. Because the overall yield of $\mathbf{1 0 b}$ using this approach was poor, an alternate strategy was adopted for synthesizing a high yield of $\mathbf{1 0 b}$.

Table 1. Synthesis of the intermediate compound $\mathbf{1 0 b}$.

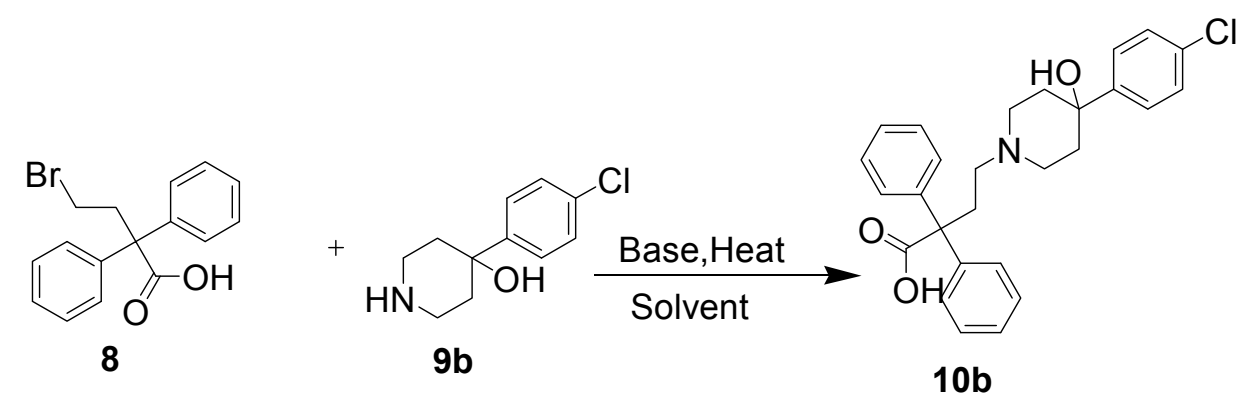

\begin{tabular}{cccc}
\hline Entry & Ratio 8:9b:DIPEA & Reaction conditions & Yield *10b \\
\hline 1 & $1: 1.5: 0$ & $\mathrm{THF}$, reflux, 2 days & - \\
2 & $1: 1: 3$ & $\mathrm{THF}$, reflux, 2 days & - \\
3 & $1: 1: 3$ & $\mathrm{CH}_{3} \mathrm{CN}, 70{ }^{\circ} \mathrm{C}, 30 \mathrm{~h}$ & - \\
4 & $1: 1: 3$ & $\mathrm{CH}_{3} \mathrm{CN}, 85^{\circ} \mathrm{C}, 30 \mathrm{~h}$ & Trace \\
5 & $1: 1: 3$ & $\mathrm{CH}_{3} \mathrm{CN}, 105^{\circ} \mathrm{C}, 30 \mathrm{~h}$ & $6 \%$ \\
\hline
\end{tabular}

* Isolated yield.

Subsequently, we designed another approach to prepare the intermediate 10b involving the alkylation of 4-(4-chlorophenyl)-4-hydroxylpiperidine with 4-bromo-2,2-diphenylbutyronitrile in the presence of DIPEA, followed by the hydrolysis of $\mathbf{1 2 b}$ with $\mathrm{KOH}$ in ${ }^{\mathrm{t}} \mathrm{BuOH}$ to afford compound $\mathbf{1 3 \mathbf { b }}$ (Scheme 1) [18]. We also prepared analogs 6 and 7 using a similar procedure for the future radiolabeling study. The slow hydrolysis of amide precursor $13 \mathbf{b}$ in $40 \% \mathrm{H}_{2} \mathrm{SO}_{4}$ aq. at reflux gave a trace amount of $\mathbf{1 0 b}$. The hydrolysis of nitrile compound $\mathbf{1 2 b}$ under different reaction conditions was also investigated (Table 2). Compound 10b was obtained in 7\% yield when the reaction was catalyzed with $\mathrm{H}_{2} \mathrm{O}_{2} / \mathrm{NaOH}$ in $\mathrm{H}_{2} \mathrm{O}$ at $80{ }^{\circ} \mathrm{C}$ for $20 \mathrm{~h}$ [19,20]. A trace amount of $\mathbf{1 0 b}$ was obtained when an inorganic base ( $2 \mathrm{M}$ sodium hydroxide) was used as a catalyst [21]. We isolated two byproducts, 14b (20\% 23\% yield) and 15b $(66 \% \sim 72 \%$ yield), when the reaction was performed under acidic conditions $\left(37 \% \mathrm{HCl}\right.$ or $\left.40 \% \mathrm{H}_{2} \mathrm{SO}_{4}\right)$ [22]. The principal reason was that the tertiary hydroxyl group on 13b was very easily eliminated under acidic conditions to form an alkene [17]. 
Scheme 1. Synthesis of the amides $6,13 \mathrm{~b}$ and 7.
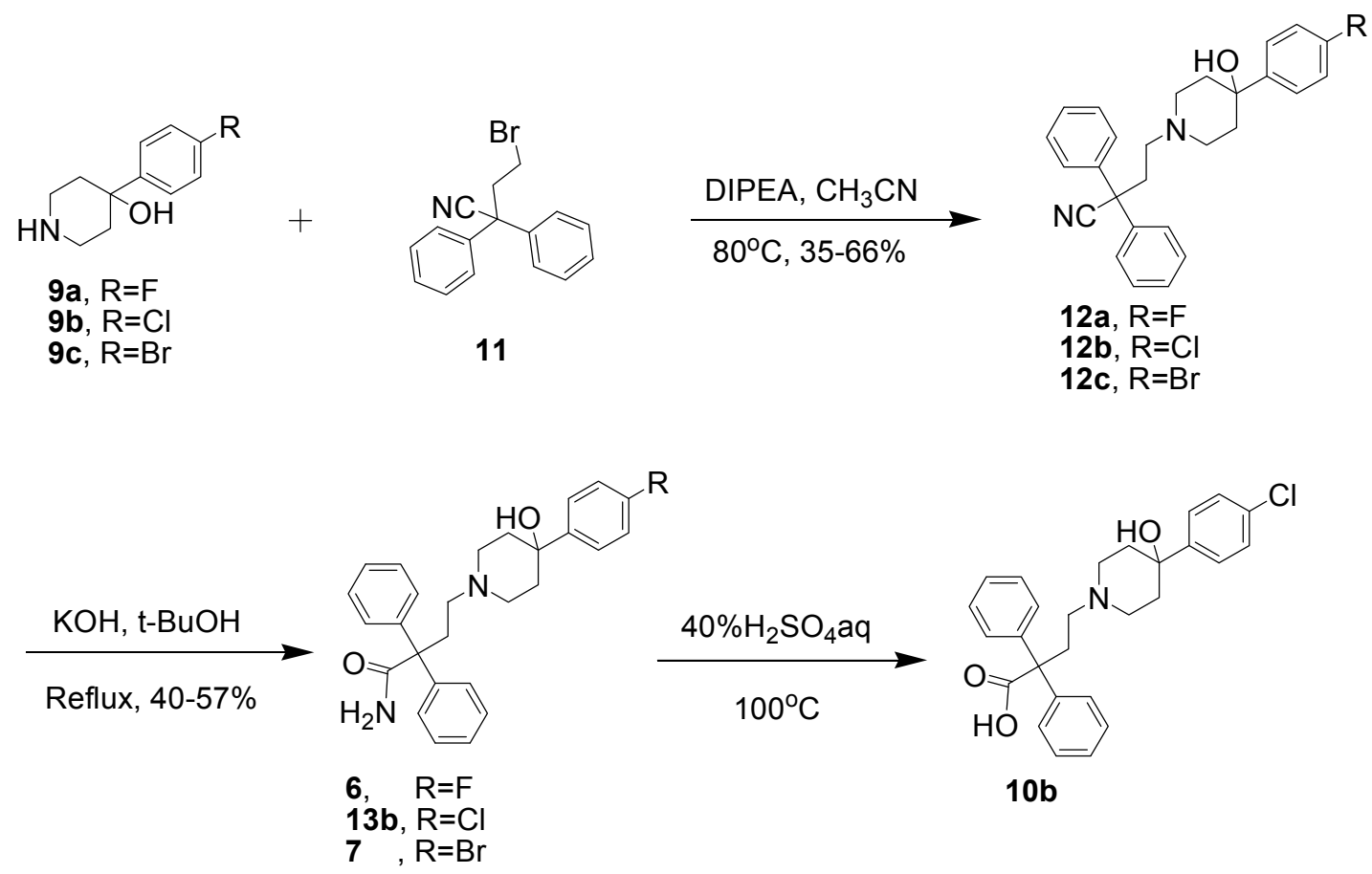

Table 2. Hydrolysis of the nitrile 12b.

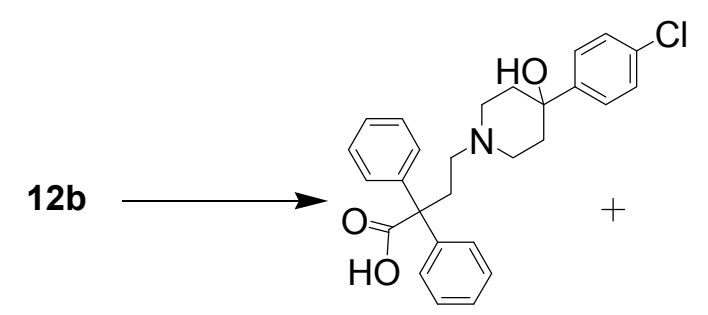

$10 \mathrm{~b}$

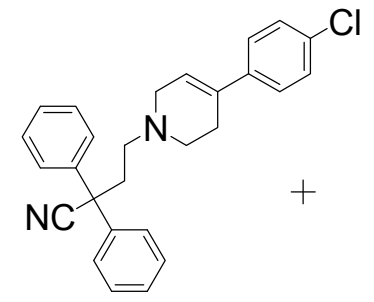

$14 \mathrm{~b}$

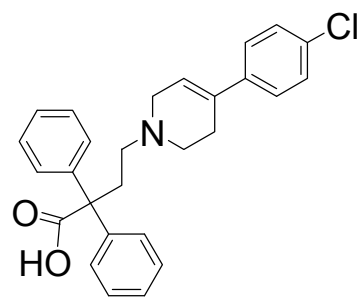

$15 b$

\begin{tabular}{|c|c|c|c|c|c|}
\hline \multirow{2}{*}{ Entry } & \multirow{2}{*}{ Catalyst } & \multirow{2}{*}{ Reaction conditions } & \multicolumn{3}{|c|}{ Yield (\%) * } \\
\hline & & & $10 \mathrm{~b}$ & $15 b$ & $14 \mathrm{~b}$ \\
\hline 1 & $\mathrm{NaOH}^{\mathrm{a}}$ & $\mathrm{CH}_{3} \mathrm{OH} / \mathrm{H}_{2} \mathrm{O}$, Reflux, $30 \mathrm{~h}$ & trace & - & - \\
\hline 2 & $\mathrm{NaOH}^{\mathrm{a}}$ & $\mathrm{H}_{2} \mathrm{O}$, Reflux, $30 \mathrm{~h}$ & trace & - & - \\
\hline 3 & $\mathrm{HCl}^{\mathrm{b}}$ & 1,4-Dioxane, Reflux, $16 \mathrm{~h}$ & - & 20 & 66 \\
\hline 4 & $\mathrm{H}_{2} \mathrm{SO}_{4}{ }^{\mathrm{c}}$ & $\mathrm{H}_{2} \mathrm{O}, 100^{\circ} \mathrm{C}, 16 \mathrm{~h}$ & - & 23 & 72 \\
\hline 5 & $\mathrm{H}_{2} \mathrm{O}_{2}{ }^{\mathrm{d}} / \mathrm{NaOH}^{\mathrm{a}}$ & $\mathrm{H}_{2} \mathrm{O}, 80 \mathrm{~h}, 20 \mathrm{~h}$ & 7 & - & - \\
\hline
\end{tabular}

Catalyst concentration: ${ }^{\mathrm{a}} 2 \mathrm{M} ;{ }^{\mathrm{b}} 37 \%{ }^{\mathrm{c}} 40 \% ;{ }^{\mathrm{d}} 30 \%$. * Isolated yield.

The desired product 5 was obtained in low yield by coupling 10b with the amine $\mathbf{9 b}$ in a reaction catalyzed by $\mathrm{TiCl}_{4}$ in toluene refluxing for $20 \mathrm{~h}$ (Scheme 2) [23]. Unfortunately, this second approach was not feasible for use in the rest of the study. 
Scheme 2. Synthesis of compound 5.

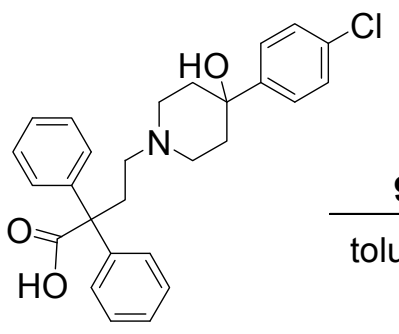

$10 \mathrm{~b}$

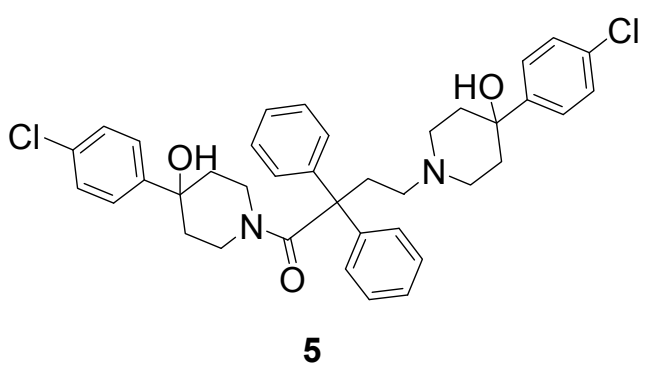

5

When the first approach was analyzed in detail, it was determined that the formation of a substantial amount of salt at the start of reaction may be responsible for the low yield of $\mathbf{1 2 b}$. Thus, a new method involving the protection of the carboxyl group of $\mathbf{8}$ with a methyl group to form an ester precursor before coupling with $\mathbf{9 b}$ was developed (Scheme 3). A mixture of 4-bromo-2,2-diphenylbutyric acid $\mathbf{8}$, thionyl chloride and catalytic DMF in dichloromethane was stirred and refluxed for $3 \mathrm{~h}$. After concentration under reduced pressure, crude 4-bromo-2,2-diphenylbutyric acid chloride (17) was achieved as a pale yellow oil. Compound $\mathbf{1 7}$ was converted into the ester $\mathbf{1 8}$ in excellent yield (82\%) by reaction with methanol at $50{ }^{\circ} \mathrm{C}$ for $4 \mathrm{~h}$. The desired product, $\mathbf{5}$, was obtained as a white solid in $71 \%$ yield by condensing 18 with $\mathbf{9 b}$ in the presence of DIPEA in $\mathrm{CH}_{3} \mathrm{CN}$ at $80{ }^{\circ} \mathrm{C}$ for $15 \mathrm{~h}$ (Scheme 3). The side product 19 was also obtained as a pale orange solid in $8 \%$ yield.

Scheme 3. The Synthesis of the ester 19 and $\mathbf{5 .}$
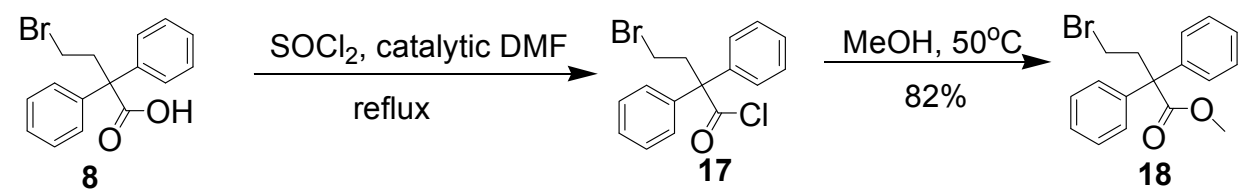

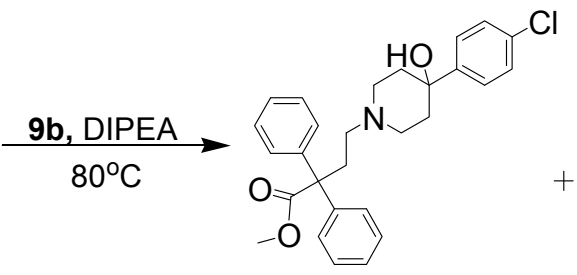

19

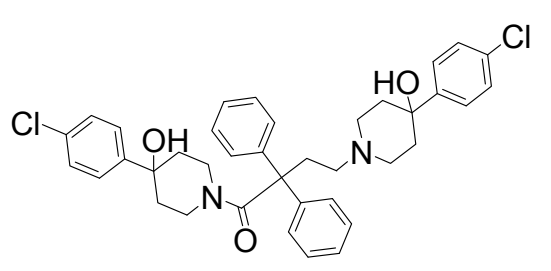

5

\section{Experimental}

\subsection{General}

All reagents and organic solvents were ACS grade or higher and used without further purification. Unless otherwise noted, all chemicals were purchased from J\&K Scientific (Shanghai, China). Reactions were performed under argon atmosphere with standard Schlenk techniques. Thin layer chromatography was performed on HAIYANG silica gel F254 plate, and compounds were visualized under UV light $(\lambda=254 \mathrm{~nm})$. Column chromatography was carried out using HAIYANG silica gel (type: $200-300$ mesh ZCX-2). ${ }^{1} \mathrm{H}(500 \mathrm{MHz}),{ }^{13} \mathrm{C}-\mathrm{NMR}(126 \mathrm{MHz})$ and ${ }^{19} \mathrm{~F}-\mathrm{NMR}(470 \mathrm{MHz})$ spectra were recorded on an Avance 500 spectrometer (Bruker; Billerica, MA, USA). Chemical shifts are reported in $\delta$ units (ppm) downfield relative to the chemical shift for tetramethylsilane. 
4-(4-(4-Chlorophenyl)-4-hydroxypiperidin-1-yl)-2,2-diphenylbutanoic acid (10b). Entry 2. The 4-bromo-2,2-diphenylbutanoic acid $8(200 \mathrm{mg}, 0.6266 \mathrm{mmol})$ was dissolved in THF (10 $\mathrm{mL})$, followed by addition of 4-(4-chlorophenyl)-piperidin-4-ol (133 mg, $0.6266 \mathrm{mmol}, 1$ eq.) and DIPEA ( $0.242 \mathrm{~mL}, 1.8798 \mathrm{mmol}, 3$ eq.) to the solution. The mixture was refluxed for 2 days. No new product was found via TLC. Entry 5. The 4-bromo-2,2-diphenylbutanoic acid 8 (400 mg, $1.253 \mathrm{mmol}$ ) was suspended in acetonitrile $(10 \mathrm{~mL})$ and DIPEA $(0.675 \mathrm{~mL}, 3.75 \mathrm{mmol})$ was added. The mixture was stirred at $105{ }^{\circ} \mathrm{C}$ for $30 \mathrm{~h}$. After the solvent was removed under vacuum, the crude was dissolved in $\mathrm{CH}_{2} \mathrm{Cl}_{2}$ and was introduced onto a silica gel column. The product was eluted with $3 \% \mathrm{MeOH}$ in dichloromethane to give $\mathbf{1 0 b}$ as a pale-orange solid (20 mg, $6 \%$ yield). TLC (silica gel; $\mathrm{MeOH}-\mathrm{CH}_{2} \mathrm{Cl}_{2}$ $\left.(5: 95 \mathrm{v}: \mathrm{v}) ; \mathrm{R}_{\mathrm{f}}=0.40\right)$. LC-MS $\left(\mathrm{M}^{+}+1\right)$ : found 449.88; calcd for $\mathrm{C}_{27} \mathrm{H}_{29} \mathrm{ClNO}_{3}, 450.18$.

4-(4-(4-Chlorophenyl)-4-hydroxypiperidin-1-yl)-2,2-diphenylbutanenitrile (12b). 4-(4-Chlorophenyl)4-hydroxypiperidine $(2.1 \mathrm{~g}, 10.0 \mathrm{mmol})$ was suspended in acetonitrile $(15 \mathrm{~mL})$ and DIPEA $(3.5 \mathrm{~mL}$, $30 \mathrm{mmol}$ ) was added. 4-Bromo-2,2-diphenylbutanenitrile (3.00 g, $10 \mathrm{mmol})$ in acetonitrile $(15 \mathrm{~mL})$ was then added. The reaction mixture was stirred at $70{ }^{\circ} \mathrm{C}$ for $30 \mathrm{~h}$. After the solvent was removed under vacuum, the crude material was redissolved in $\mathrm{CH}_{2} \mathrm{Cl}_{2}$ and introduced onto a silica gel column. The product was eluted with $5 \% \mathrm{MeOH}$ in dichloromethane to yield $\mathbf{1 2 b}$ as a pale-orange solid (2.8 g, $66 \%$ yield). TLC (silica gel; $\left.\mathrm{MeOH}-\mathrm{CH}_{2} \mathrm{Cl}_{2}(5: 95 \mathrm{v}: \mathrm{v}) ; \mathrm{R}_{\mathrm{f}}=0.50\right) .{ }^{1} \mathrm{H}-\mathrm{NMR}\left(\mathrm{CDCl}_{3}\right): \delta 7.37$ (m, $14 \mathrm{H}), 2.80(\mathrm{~d}, 2 \mathrm{H}, J=11.25 \mathrm{~Hz}), 2.67(\mathrm{~m}, 2 \mathrm{H}), 2.55(\mathrm{~m}, 2 \mathrm{H}), 2.47(\mathrm{t}, 2 \mathrm{H}, J=11.3 \mathrm{~Hz}), 2.105(\mathrm{~m}, 2 \mathrm{H})$, $1.73(\mathrm{~d}, 2 \mathrm{H}, J=11.9 \mathrm{~Hz}) .{ }^{13} \mathrm{C}-\mathrm{NMR}(\mathrm{CDCl} 3): \delta 140.16,132.95,129.09,128.57,128.10,126.95$, 126.23, 122.27, 71.11, 54.93, 50.17, 49.71, 38.52, 36.80ppm. LC-MS $\left(\mathrm{M}^{+}+1\right)$ found 431.20; calcd for $\mathrm{C}_{27} \mathrm{H}_{28} \mathrm{ClN}_{2} \mathrm{O}, 431.19$.

4-(4-(4-Fluorophenyl)-4-hydroxypiperidin-1-yl)-2,2-diphenylbutanenitrile (12a). Compound 12a was synthesized using the same procedure that was followed the synthesis of the compound $\mathbf{1 2 b}$, and was obtained in $35 \%$ yield as a pale-orange solid. ${ }^{1} \mathrm{H}-\mathrm{NMR}\left(\mathrm{CDCl}_{3}\right): \delta 7.45(\mathrm{~m}, 2 \mathrm{H}), 7.39(\mathrm{~d}, 4 \mathrm{H}, J=10 \mathrm{~Hz})$, $7.35(\mathrm{t}, 4 \mathrm{H}, J=5 \mathrm{~Hz}), 7.29(\mathrm{t}, 2 \mathrm{H}, J=10 \mathrm{~Hz}), 7.00(\mathrm{t}, 2 \mathrm{H}, J=10 \mathrm{~Hz}), 2.75(\mathrm{~d}, 2 \mathrm{H}, J=10 \mathrm{~Hz}), 2.63$ $(\mathrm{m}, 2 \mathrm{H}), 2.52(\mathrm{~m}, 2 \mathrm{H}), 2.47(\mathrm{t}, 2 \mathrm{H}, J=10 \mathrm{~Hz}), 2.08(\mathrm{~m}, 2 \mathrm{H}), 1.71(\mathrm{~d}, 2 \mathrm{H}, J=15 \mathrm{~Hz}) .{ }^{13} \mathrm{C}-\mathrm{NMR}: \delta$ $162.89,160.93,144.27,140.16,129.03,128.05,126.91,126.45,126.39,122.23,115.18,115.01$, 70.91, 54.88, 50.19, 49.72, 38.56, 36.74. ${ }^{19}$ F-NMR: $\delta$ 116.12. LC-MS $\left(\mathrm{M}^{+}+1\right)$ : Found: 415.06; Calcd: $\mathrm{C}_{27} \mathrm{H}_{28} \mathrm{FN}_{2} \mathrm{O}: 415.22$.

4-(4-(4-Bromophenyl)-4-hydroxypiperidin-1-yl)-2,2-diphenylbutanenitrile (12c). Compound 12c was synthesized using the same procedure that was followed the synthesis of the compound $\mathbf{1 2 b}$, and was obtained in 58\% yield as a pale-orange solid. ${ }^{1} \mathrm{H}-\mathrm{NMR}\left(\mathrm{CDCl}_{3}\right): \delta 7.48(\mathrm{~d}, 2 \mathrm{H}, J=10 \mathrm{~Hz}), 7.41(\mathrm{~d}, 4 \mathrm{H}$, $J=10 \mathrm{~Hz}), 7.37(\mathrm{~m}, 6 \mathrm{H}), 7.31(\mathrm{t}, 2 \mathrm{H}, J=5 \mathrm{~Hz}), 2.76(\mathrm{~d}, 2 \mathrm{H}, J=10 \mathrm{~Hz}), 2.65(\mathrm{~m}, 2 \mathrm{H}), 2.54(\mathrm{~m}, 2 \mathrm{H})$, $2.45(\mathrm{t}, 2 \mathrm{H}, J=10 \mathrm{~Hz}), 2.08(\mathrm{~m}, 2 \mathrm{H}), 1.68(\mathrm{~m}, 3 \mathrm{H}) .{ }^{13} \mathrm{C}-\mathrm{NMR}: \delta$ 147.64, 140.11, 131.47, 129.07, 128.09, 126.91, 126.66, 122.25, 121.00, 70.97, 54.90, 50.18, 49.64, 38.35, 36.67. LC-MS $\left(\mathrm{M}^{+}+1\right)$ : Found: 476.92; Calcd: $\mathrm{C}_{27} \mathrm{H}_{28} \mathrm{BrN}_{2} \mathrm{O}: 477.13$.

4-(4-(4-Chlorophenyl)-4-hydroxypiperidin-1-yl)-2,2-diphenylbutanamide

(13b). 4-(4-(4-Chlorophenyl)-4-hydroxypiperidin-1-yl)-2,2-diphenylbutanenitrile (2.5 g, $6.0 \mathrm{mmol})$ was dissolved in $\mathrm{BuOH}$ $(40 \mathrm{~mL})$ and potassium hydroxide $(1.2 \mathrm{~g}, 21.3 \mathrm{mmol})$ was added. The reaction mixture was stirred at 
$100{ }^{\circ} \mathrm{C}$ for $3 \mathrm{~d}$. After concentration under vacuum, the crude material was redissolved in dichloromethane and filtered through a pad of Celite. Chromatography of the sample on a silica gel column eluted with ammonium hydroxide $(2 \mathrm{M})$ solution in $\mathrm{MeOH}-\mathrm{CH}_{2} \mathrm{Cl}_{2}(5: 95 \mathrm{v} / \mathrm{v})$ gave $\mathbf{1 3 b}$ as a pale-yellow solid (1.58 g, 55\% yield). TLC (silica gel; $\left.\mathrm{MeOH}-\mathrm{CH}_{2} \mathrm{Cl}_{2}(5: 95 \mathrm{v}: \mathrm{v}) ; \mathrm{R}_{\mathrm{f}}=0.40\right) .{ }^{1} \mathrm{H}-\mathrm{NMR}$ $\left(\mathrm{CDCl}_{3}\right): \delta 7.36(\mathrm{~m}, 14 \mathrm{H}), 6.58(\mathrm{~s}, 1 \mathrm{H}), 5.46(\mathrm{~s}, 1 \mathrm{H}), 3.91(\mathrm{~s}, 1 \mathrm{H}), 2.83(\mathrm{~m}, 2 \mathrm{H}), 2.69(\mathrm{~s}, 2 \mathrm{H}), 2.39(\mathrm{~m}$, $3 \mathrm{H}), 2.10(\mathrm{~m}, 2 \mathrm{H}), 1.74(\mathrm{~d}, 2 \mathrm{H}, J=12.85 \mathrm{~Hz}) .{ }^{13} \mathrm{C}-\mathrm{NMR}: 176.56,167.25,158.96,146.67,143.26$, 132.82, 128.69, 128.41, 127.06, 126.10, 59.91, 54.92, 49.48, 38.28, 35.87. LC-MS $\left(\mathrm{M}^{+}+1\right)$ : Found: 449.3; Calcd: $\mathrm{C}_{27} \mathrm{H}_{30} \mathrm{ClN}_{2} \mathrm{O}_{2}$ : 449.20.

4-(4-(4-Fluorophenyl)-4-hydroxypiperidin-1-yl)-2,2-diphenylbutanamide (6). Compound 6 was synthesized using the same procedure that was followed the synthesis of the compound $\mathbf{1 3 b}$, and was obtained in $40 \%$ yield as a pale-orange solid. ${ }^{1} \mathrm{H}-\mathrm{NMR}\left(\mathrm{CDCl}_{3}\right): \delta 7.45(\mathrm{~m}, 2 \mathrm{H}), 7.31(\mathrm{~m}, 10 \mathrm{H}), 6.99$ (t, $2 \mathrm{H}, J=10 \mathrm{~Hz}), 5.07(\mathrm{~s}, 1 \mathrm{H}), 2.89(\mathrm{~m}, 2 \mathrm{H}), 2.64(\mathrm{~m}, 2 \mathrm{H}), 2.35(\mathrm{~m}, 2 \mathrm{H}), 2.04(\mathrm{~m}, 2 \mathrm{H}), 1.59(\mathrm{~d}, 2 \mathrm{H}$, $J=5 \mathrm{~Hz}), 6.22(\mathrm{~s}, 1 \mathrm{H}), 5.82(\mathrm{~s}, 1 \mathrm{H}), 3.02(\mathrm{~m}, 2 \mathrm{H}), 2.82(\mathrm{~m}, 4 \mathrm{H}), 2.63(\mathrm{~m}, 2 \mathrm{H}), 2.36(\mathrm{~m}, 2 \mathrm{H}), 1.76(\mathrm{~d}$, $2 \mathrm{H}, J=10 \mathrm{~Hz}) .{ }^{13} \mathrm{C}-\mathrm{NMR}: \delta 175.53,162.43,160.51,145.88,143.83,129.27,128.46,127.29,127.24$, 127.07, 115.15, 114.98, 69.28, 59.54, 54.73, 49.44, 37.33, 29.55. ${ }^{19}$ F-NMR: $\delta 117.01$.

4-(4-(4-Bromophenyl)-4-hydroxypiperidin-1-yl)-2,2-diphenylbutanamide (7). Compound 7 was synthesized using the same procedure that was followed the synthesis of the compound $\mathbf{1 3 \mathbf { b }}$, and was obtained in 57\% yield as a pale-orange solid. ${ }^{1} \mathrm{H}-\mathrm{NMR}\left(\mathrm{CDCl}_{3}\right): \delta 7.41(\mathrm{t}, 2 \mathrm{H}, J=5 \mathrm{~Hz}), 7.31(\mathrm{~m}$, $12 \mathrm{H}), 6.45(\mathrm{~s}, 1 \mathrm{H}), 5.87(\mathrm{~s}, 1 \mathrm{H}), 2.82(\mathrm{~m}, 12 \mathrm{H}), 2.31(\mathrm{~m}, 12 \mathrm{H}), 2.50(\mathrm{~m}, 12 \mathrm{H}), 2.42(\mathrm{~m}, 12 \mathrm{H}), 2.11(\mathrm{~m}$, 12H), 1.69 (m, 12H). ${ }^{13} \mathrm{C}-\mathrm{NMR}: \delta 176.79,147.17,143.14,131.46,128.75,128.57,127.26,126.64$, 121.06, 70.67, 59.88, 55.00, 49.48, 37.79, 35.46. LC-MS $\left(\mathrm{M}^{+}+1\right)$ : Found: 494.93; Calcd: $\mathrm{C}_{27} \mathrm{H}_{30} \mathrm{BrN}_{2} \mathrm{O}_{2}^{+}: 495.14$.

4-(4-(4-Chlorophenyl)-4-hydroxypiperidin-1-yl)-2,2-diphenylbutanoic acid (10b). 4-(4-(4-Chlorophenyl)-4-hydroxycyclohexyl)-2,2-diphenylbutanamide (1.2 g, $2.6 \mathrm{mmol})$ was added to $40 \% \mathrm{H}_{2} \mathrm{SO}_{4}$ $(50 \mathrm{~mL})$ and the mixture was stirred at $100{ }^{\circ} \mathrm{C}$ for 2 days. The crude material was extracted with ethyl acetate $(60 \mathrm{~mL} \times 3)$. The ethyl acetate was removed by reduced pressure distillation. After drying with $\mathrm{MgSO}_{4}$ and dissolving in $\mathrm{CH}_{2} \mathrm{Cl}_{2}(2 \mathrm{~mL})$, the product was introduced onto a silica gel and eluted with $5 \% \mathrm{MeOH}$ in $\mathrm{CH}_{2} \mathrm{Cl}_{2}$ to give trance amount of 10b. TLC (silica gel; $\mathrm{MeOH}-\mathrm{CH}_{2} \mathrm{Cl}_{2}$ (5:95 v:v); $\mathrm{R}_{\mathrm{f}}=0.30$ ). ${ }^{1} \mathrm{H}-\mathrm{NMR}\left(\mathrm{CDCl}_{3}\right): \delta 7.3096(\mathrm{~m}, 14 \mathrm{H}), 5.9319(\mathrm{~s}, 1 \mathrm{H}), 3.29(\mathrm{~m}, 2 \mathrm{H}), 2.94(\mathrm{~m}, 2 \mathrm{H}), 2.80(\mathrm{~m}, 2 \mathrm{H}), 2.72$ (m, 2H), 2.67 (m, 4H), 2.10 (m, 2H). LC-MS (M+1): found: 449.88; Calcd for $\mathrm{C}_{27} \mathrm{H}_{28} \mathrm{ClNO}_{3}{ }^{+}, 450.18$.

\section{Procedure for Preparing $\mathbf{1 0 b}$ from $\mathbf{1 2 b}$}

Entry 3. 4-(4-(4-Chlorophenyl)-4-hydroxypiperidine-1-yl)-2,2-diphenylbutanenitrile (400 mg, $0.928 \mathrm{mmol})$ was dissolved in 1,4-dioxane $(10 \mathrm{~mL})$ and $37 \%$ hydrochloric acid $(5 \mathrm{~mL})$ was then added. The reaction mixture was refluxed for $16 \mathrm{~h}$. After concentration under vacuum, the crude was dissolved in trichloromethane $(10 \mathrm{~mL})$ and extracted with $\mathrm{H}_{2} \mathrm{O}(20 \mathrm{~mL} \times 3)$. After the organic layer was dried by $\mathrm{MgSO}_{4}$, the solvent was removed under vacuum and redissolved in $\mathrm{CH}_{2} \mathrm{Cl}_{2}$. The mixture was introduced onto a silica gel column and the product was eluted with 50\% EtOAc in hexane first and $5 \% \mathrm{MeOH}$ in $\mathrm{CH}_{2} \mathrm{Cl}_{2}$ later to give the product $\mathbf{1 4 b}$ as a yellow solid (250 mg, 66\%). TLC (silica gel; 
EtOAc-hexane $\left.(1: 1 \mathrm{v}: \mathrm{v}) ; \mathrm{R}_{\mathrm{f}}=0.70\right)$. Compound 14b: ${ }^{1} \mathrm{H}-\mathrm{NMR}\left(\mathrm{CDCl}_{3}\right): \delta 7.36(\mathrm{~m}, 14 \mathrm{H}), 6.04(\mathrm{~m}$, $1 \mathrm{H}), 3.16(\mathrm{~m}, 2 \mathrm{H}), 2.74(\mathrm{~m}, 2 \mathrm{H}), 2.62(\mathrm{~m}, 4 \mathrm{H}), 2.53(\mathrm{~m}, 2 \mathrm{H}) \mathrm{ppm} .{ }^{13} \mathrm{C}-\mathrm{NMR}\left(\mathrm{CDCl}_{3}\right): \delta 141.44$, $140.57,135.56,134.26,130.53,129.92,129.55,128.27,127.72,123.52,55.96,54.81,51.93,51.57$, 38.48, 29.44 ppm. MS (M+1): found 412.99; Calcd $\mathrm{C}_{27} \mathrm{H}_{25} \mathrm{ClN}_{2}^{+}, 412.95$.

1,4-bis(4-(4-Chlorophenyl)-4-hydroxypiperidin-1-yl)-2,2-diphenylbutan-1-one

(5). 4-(4-Chlorophenyl)-4-hydroxypiperidinorophenyl)-4-hydroxypiperidine (28 mg, $0.12 \mathrm{mmol}, 2$ eq.) was dissolved in toluene $(5 \mathrm{~mL})$ and 4-(4-(4-chlorophenyl)-4- hydroxypiperidin-1-yl)-2,2-diphenylbutanoic acid (30 mg, $0.067 \mathrm{mmol})$ and $\mathrm{TiCl}_{4}(1.5 \mathrm{~mL})$ was then added. The mixture was refluxed for $20 \mathrm{~h}$. After the solvent was removed under vacuum, the crude material was dissolved in $\mathrm{CH}_{2} \mathrm{Cl}_{2}(10 \mathrm{~mL})$. After the mixture was filtered and $\mathrm{CH}_{2} \mathrm{Cl}_{2}$ was then removed to $2 \mathrm{~mL}$, the product was introduced onto a silica gel with $5 \% \mathrm{MeOH}$ in $\mathrm{CH}_{2} \mathrm{Cl}_{2}$ to give 5 as a pale-orange solid (trace amount). TLC (silica gel column; $\left.\mathrm{MeOH}-\mathrm{CH}_{2} \mathrm{Cl}_{2}(5: 95 \mathrm{v}: \mathrm{v}) ; \mathrm{R}_{\mathrm{f}}=0.40\right)$.

Methyl 4-bromo-2,2-diphenylbutanoate (18). 4-Bromo-2,2-diphenylbutanoic acid (2.0 g, $6.266 \mathrm{mmol})$ was dissolved in dry $\mathrm{CH}_{2} \mathrm{Cl}_{2}(20 \mathrm{~mL})$ and thionyl chloride $(2.27 \mathrm{~mL}, 31.33 \mathrm{mmol}, 5$ eq. $)$ was then added slowly. A trace amount of DMF was added later. The reaction mixture was refluxed at $\mathrm{N}_{2}$ for $3 \mathrm{~h}$. Methyl alcohol ( $2 \mathrm{~mL}$, excess) was added slowly and the mixture was stirred at $50{ }^{\circ} \mathrm{C}$ for $3 \mathrm{~h}$. After the solvent was removed under vacuum, the crude material was redissolved in $\mathrm{CH}_{2} \mathrm{Cl}_{2}$ and introduced onto a silica gel column. The product was eluted with $10 \% \mathrm{EA}$ in hexane to give a pale-orange oil (900 $\mathrm{mg}$, $43 \%$ yield).TLC (silica gel; EtAc-hexane (10:90 v:v); $\left.\mathrm{R}_{\mathrm{f}}=0.80\right) .{ }^{1} \mathrm{H}-\mathrm{NMR}\left(\mathrm{CDCl}_{3}\right): \delta 7.31(\mathrm{~m}, 10 \mathrm{H})$, $3.74(\mathrm{~s}, 1 \mathrm{H}), 3.13(\mathrm{~m}, 2 \mathrm{H}), 3.00(\mathrm{~m}, 2 \mathrm{H}) .{ }^{13} \mathrm{C}-\mathrm{NMR}\left(\mathrm{CDCl}_{3}\right): \delta 174.05,141.70,128.70,128.42,127.46$, 60.84, 52.78, 42.0447, 29.18 ppm. GC-MS: found: 331.99; Calcd for $\mathrm{C}_{17} \mathrm{H}_{17} \mathrm{BrO}_{2}$, 332.04.

Methyl4-(4-(4-chlorophenyl)-4-hydroxypiperidin-1-yl)-2,2-diphenylbutanoate (19) and 1,4-bis(4-(4chlorophenyl)-4-hydroxypiperidin-1-yl)-2,2-diphenyl -butan-1-one (5). Methyl 4-bromo-2,2diphenylbutanoate $(800 \mathrm{mg}, 2.4 \mathrm{mmol})$ was dissolved in $\mathrm{CH}_{3} \mathrm{CN}$ (15 mL) and 4-(4-chlorophenyl)-4hydroxypiperidine (406 mg, $1.92 \mathrm{mmol}, 0.8 \mathrm{eq}$.) was then added. The reaction mixture was stirred at $50{ }^{\circ} \mathrm{C}$ for $30 \mathrm{~h}$. After concentration under vacuum, the crude material was redissolved in $\mathrm{CH}_{2} \mathrm{Cl}_{2}$ and introduced onto a silica gel column. The product was eluted with $3 \% \mathrm{MeOH}$ in $\mathrm{CH}_{2} \mathrm{Cl}_{2}$ first and $5 \%$ $\mathrm{MeOH}$ in $\mathrm{CH}_{2} \mathrm{Cl}_{2}$ later to get $\mathbf{1 9}$ as a pale-orange solid (70 mmg, $8 \%$ yield) and 20 as a pale-orange solid (437 mg, 71\%). Compound 19: TLC (silica gel; $\left.\mathrm{MeOH}-\mathrm{CH}_{2} \mathrm{Cl}_{2}(10: 90 \mathrm{v}: \mathrm{v}) ; \mathrm{R}_{\mathrm{f}}=0.40\right)$. ${ }^{1} \mathrm{H}-\mathrm{NMR}$ $\left(\mathrm{CDCl}_{3}\right): \delta 7.34(\mathrm{~m}, 14 \mathrm{H}), 3.74(\mathrm{~s}, 3 \mathrm{H}), 3.28(2 \mathrm{H}, \mathrm{m}), 3.13(2 \mathrm{H}, \mathrm{m}), 3.12(2 \mathrm{H}, \mathrm{m}), 3.10(4 \mathrm{H}, \mathrm{m}), 2.60$ $(1 \mathrm{H}, \mathrm{s}), 2.06(2 \mathrm{H}, \mathrm{d}, J=14.0 \mathrm{~Hz}) .{ }^{13} \mathrm{C}-\mathrm{NMR}\left(\mathrm{CDCl}_{3}\right): \delta 173.06,143.62,140.18,132.54,127.72$, 127.52, 127.28, 126.62, 126.32, 125.04, 68.58, 58.02, 51.81, 48.22, 34.51, 31.73, 30.93, 28.36, 21.69. MS: found: 463.92; calcd for $\mathrm{C}_{28} \mathrm{H}_{31} \mathrm{ClNO}_{3}{ }^{+}$, 464.20. Compound 5: TLC (silica gel; $\mathrm{MeOH}-\mathrm{CH}_{2} \mathrm{Cl}_{2}$ $\left.(10: 90 \mathrm{v}: \mathrm{v}) ; \mathrm{R}_{\mathrm{f}}=0.40\right) .{ }^{1} \mathrm{H}-\mathrm{NMR}\left(\mathrm{CDCl}_{3}\right): \delta 7.3094(\mathrm{~m}, 18 \mathrm{H}), 4.5945(\mathrm{~d}, 1 \mathrm{H}, J=11.4 \mathrm{~Hz}), 3.31(\mathrm{~m}$, $10 \mathrm{H}), 2.73(\mathrm{~m}, 2 \mathrm{H}), 2.55(\mathrm{~m}, 2 \mathrm{H}), 2.28(\mathrm{~m}, 2 \mathrm{H}), 1.87(\mathrm{~m}, 4 \mathrm{H}), 1.65(\mathrm{~m}, 2 \mathrm{H}) .{ }^{13} \mathrm{C}-\mathrm{NMR}\left(\mathrm{CDCl}_{3}\right)$ : $171.38,146.46,145.29,141.45,136.64,133.00,132.68,129.45,125.03,128.89,128.45,128.31$, $128.17,127.57,126.54,126.08,71.10,69.06,60.03,54.44,44.25,42.68,39.65,39.21,37.91,35.95$, 35.30, 29.77 ppm. MS: found: 644.88; Calcd for $\mathrm{C}_{38} \mathrm{H}_{42} \mathrm{Cl}_{2} \mathrm{~N}_{2} \mathrm{O}_{3}{ }^{+}, 644.25$. 


\section{Conclusions}

In summary, compound 5 was synthesized in excellent yield. Analogs 6 and 7 were obtained through the hydrolysis of the nitrile group in good yield. These methods have the advantages of high yields, convenient procedures, and mild reaction conditions.

\section{Supplementary Material}

Supplementary data associated with this article can be found in the online version $\left({ }^{1} \mathrm{H}-\mathrm{NMR}\right.$, ${ }^{13} \mathrm{C}-\mathrm{NMR}$, and LC-MS for all compounds), which can be accessed at: http://www.mdpi.com/14203049/17/12/14288/s1.

\section{Acknowledgments}

This research was supported by Jiangsu Science Foundation BK2011704 and NJUST Research Funding (NO. 2011ZDJH08).We thank Wei Jiang for helpful experimental suggestions.

\section{References}

1. Davis, M.P. Evidence from Basic Research for Opioid Combinations. Expert Opin. Drug Discov. 2012, 7, 165-178.

2. Chevlen, E. Opioids: A review. Curr. Pain Headache Rep. 2003, 7, 15-23.

3. Lord, J.A.; Waterfield, A.A.; Hughes, J.; Kosterlitz, H.W. Endogenous Opioid Peptides: Multiple Agonists and Receptors. Nature 1977, 267, 495-499.

4. Pasternak, G.W. Multiple Opiate Receptors: $\left[{ }^{3} \mathrm{H}\right]$-Ethylketocyclazocine Receptor Binding and Ketocyclazocine Analgesia. Proc. Natl. Acad. Sci. USA 1980, 77, 3691-3694.

5. Tokuyama, S.; Inoue, M.; Fuchigami, T.; Ueda, H. Lack of Tolerance in Peripheral Opioid Analgesia in Mice. Life Sci. 1998, 62, 1677-1681.

6. Bigliardi, P.L.; Bigliardi-Qi, M.; Buechner, S.; Rufli, T. Expression of mu-Opiate Receptor in Human Epidermis and Keratinocytes. J. Invest. Dermatol. 1998, 111, 297-301.

7. Decker, M.; Fulton, B.F.; Zhang, B.; Knapp, B.I.; Bidlack, J.M.; Neumeyer, J.L. Univalent and Bivalent Ligands of Butorphan: Characteristics of the Linking Chain Determine the Affinity and Potency of Such Opioid Ligands. J. Med. Chem. 2009, 52, 7389-7396.

8. Peng, X.; Knapp, B.I.; Bidlack, J.M.; Neumeyer, J.L. Synthesis and Preliminary in Vitro Investigation of Bivalent Ligands Containing Homo- and Heterodimeric Pharmacophores at mu, delta, and kappa Opioid Receptors. J. Med. Chem. 2006, 49, 256-262.

9. Awouters, F.; Megens, A.; Verlinden, M.; Schuurkes, J.; Niemegeers, C.; Janssen, P.A. Loperamide: Survey of Studies on Mechanism of its Antidiarrheal Activity. Dig. Dis. Sci. 1993, 38, 977-995.

10. Sato, S.; Komoto, T.; Kanamaru, Y.; Kawamoto, N.; Okada, T.; Kaiho, T.; Mogi, K.; Morimoto, S.; Umehara, N.; Koda, T.; et al. New mu-Opioid Receptor Agonists with Phenoxyacetic Acid Moiety. Chem. Pharm. Bull. 2002, 50, 292-297.

11. Chen, Z.; Miller, W.; Shan, S.; Valenzano, K. Design and parallel synthesis of piperidine libraries targeting the nociceptin (N/OFQ) receptor. Bioorg. Med. Chem. Lett. 2003, 13, 3247-3252. 
12. Komoto, T.; Okada, T.; Sato, S.; Niino, Y.; Oka, T.; Sakamoto, T. New mu-Opioid Receptor Agonists with Piperazine Moiety. Chem. Pharm. Bull. 2001, 49, 1314-1320.

13. Valenzano, K.J.; Miller, W.; Chen, Z.; Shan, S.; Crumley, G.; Victory, S.F.; Davies, E.; Huang, J.C.; Allie, N.; Nolan, S.J.; et al. DiPOA ([8-(3,3-diphenylpropyl)-4-oxo-1-phenyl-1,3,8triazaspiro[4.5]dec-3-yl]-acetic acid), a Novel, Systemically Available, and Peripherally Restricted $\mathrm{Mu}$ Opioid Agonist with Antihyperalgesic Activity: II. In Vivo Pharmacological Characterization in the Rat. J. Pharmacol. Exp. Ther. 2004, 310, 793-799.

14. Valenzano, K.J.; Miller, W.; Chen, Z.; Shan, S.; Crumley, G.; Victory, S.F.; Davies, E.; Huang, J.C.; Allie, N.; Nolan, S.J.; et al. DiPOA ([8-(3,3-Diphenylpropyl)-4-oxo-1-Phenyl-1,3,8-Triazaspiro[4.5]Dec-3-yl]Acetic Acid), A Novel, Systemically Available, and Peripherally Restricted mu Opioid Agonist with Antihyperalgesic Activity: I. In Vitro Pharmacological Characterization and Pharmacokinetic Properties. J. Pharmacol. Exp. Ther. 2004, 310, 783-792.

15. Di Bosco, A.M.; Grieco, P.; Diurno, M.V.; Campiglia, P.; Novellino, E.; Mazzoni, O. Binding Site of Loperamide: Automated Docking of Loperamide in Human mu- and delta-opioid receptors. Chem. Biol. Drug Des. 2008, 71, 328-335.

16. Khan, P.M.; Correa, R.G.; Divlianska, D.B.; Peddibhotla, S.; Sessions, E.H.; Magnuson, G.; Brown, B.; Suyama, E.; Yuan, H.; Mangravita-Novo, A.; et al. Identification of Inhibitors of NOD1-Induced Nuclear Factor-kB Activation. ACS Med. Chem. Lett. 2011, 2, 780-785.

17. Bao, X.; Lu, S.; Simeon, F.G.; Victor, W.P. Synthesis of a Prospective ${ }^{18}$ F-labeled Tracer for Imaging P-Glycoprotein Function. J. Labelled Comp. Radiopharm. 2009, 52, S350.

18. Lazarova, N.; Zoghbi, S.S.; Hong, J.; Seneca, N.; Tuan, E.; Gladding, R.L.; Liow, J.S.; Taku, A.; Innis, R.B.; Pike, V.W. Synthesis and Evaluation of $\left[N\right.$-methyl- $\left.{ }^{11} \mathrm{C}\right] N$-Desmethyl-loperamide as a New and Improved PET Radiotracer for Imaging P-gp Function. J. Med. Chem. 2008, 51, 6034-6043.

19. Chen, A.; Cauchon, E.; Chefson, A.; Dolman, S.; Ducharme, Y.; Dubé, D.; Falgueyret, J.P.; Fournier, P.A.; Gagné, S.; Gallant, M.; et al. Renin inhibitors for the treatment of hypertension: Design and optimization of a novel series of tertiary alcohol-bearing piperidines. Bioorg. Med. Chem. Lett. 2011, 21, 3976-3981.

20. Pàmies, O.; Bäckvall, J.E. Efficient Lipase-Catalyzed Kinetic Resolution and Dynamic Kinetic Resolution of $\beta$-Hydroxy Nitriles. Correction of Absolute Configuration and Transformation to Chiral $\beta$-Hydroxy Acids and $\gamma$-Amino Alcohols. Adv. Synth. Catal. 2002, 344, 947-952.

21. Raparti, V.; Chitre, T.; Bothara, K.; Danqre, S.; Khachane, C.; Gore, S.; Deshmane, B. Novel 4-(morpholin-4-yl)-N'-(arylidene)benzohydrazides: Synthesis, Antimycobacterial Activity and Qsar Investigations. Eur. J. Med. Chem. 2009, 44, 3954-3960.

22. Einsiedel, J.; Schoerner, C; Gmeiner, P. Synthesis of Dihydrooxazole Analogues Derived from Linezolid. Tetrahedron 2003, 59, 3403-3407.

23. Allen, C.L.; Chhatwal, A.R.; Williams, J.M. Direct Amide Formation from Unactivated Carboxylic Acids and Amines. Chem. Commun. 2012, 48, 666-668.

Sample Availability: Samples of the compounds 5-7, 10b, 12a-c, 13b, 14b, 18 and 19 are available from the authors.

(C) 2012 by the authors; licensee MDPI, Basel, Switzerland. This article is an open access article distributed under the terms and conditions of the Creative Commons Attribution license (http://creativecommons.org/licenses/by/3.0/). 